\title{
Modeling an improved method fOr double mOdulation PhOto REFLECTANCE EXPERIMENTS
}

\author{
M. Hernández ${ }^{1} \&$ R. Ivanov. ${ }^{2}$ \\ ${ }^{1}$ Facultad de Física, UH, Cuba \\ Email: mhernan@ff.oc.uh.cu \\ ${ }^{2}$ Facultad de Física, UAZ, México \\ Email: rumen@ahobon.reduaz.mx
}

Received: November $27^{\text {st }}, 2001$. Accepted October $15^{\text {th }}, 2002$

\begin{abstract}
This paper describes an improved method to be used in double modulation photo reflectance experiments, in order to reduce the influence of parasitic effects in the useful signal. The method uses the inner and outer sections of a single chopper disk to modulate the monochromator and the laser beam, eliminating the need of complex electronic filters, and two additional PIN photodiodes for scattered light compensation. The method has been numerically simulated using LabVIEW in order to investigate the influence of the modulator finite switching time and the finite resolution of the digital acquisition system. Results show that a relative error less than $1 \%$ can be achieved.
\end{abstract}

KEYWORDS: Photo reflectance, Double modulation.

\section{INTRODUCTION}

Photo reflectance is a useful experimental technique for obtaining data on transition energies, doping levels, and quality of materials in epitaxial structures. This technique is based on the internal electric-field dependence of reflectance in semiconductor materials. The advantage of the photo reflectance method resides in it is contact less, nondestructive and simple.

The principle of photo reflectance is to measure the change in the reflection coefficient of a sample under research for several wavelengths of incident, light coming from a monochromator [1, 2]. The changes in the reflection coefficient are induced by photo injection of electron-hole pairs, generated when the sample surface is illuminated by a laser beam modulated in a mechanical chopper.

Several parasitic factors influence the results obtained in photo reflectance experiments. The most important are the scattered laser light and the photoluminescence induced in the sample when shinned by the two beams [3,4]. When both beams are modulated, as in the paper from Ghosh and Arora [5], an additional factor is present -the modulated monochromator beam.

This paper proposes an improved method referred to [5], to carry out photo reflectance experiments. To better understand the advantages of these improvements, let us describe briefly the early method used by both authors.

Vol. 2 No. 1 April 2004 
In their method, the sample under study is shinned by two beams modulated by individual choppers; $f_{1}$ is the laser beam modulation frequency and $\mathrm{f}_{2}$ is the monochromator modulation frequency. The useful signal on the PIN diode, with frequency $f_{1}+f_{2}$ is amplified and detected in a lock-in amplifier synchronized to the sum frequency.

A detailed analysis of their method, shows the following two inconvenients:

1. Due to the fact that each beam is modulated with an individual chopper, a complex electronic circuit to obtain a signal with frequency $f_{1}+f_{2}$ is necessary.

2. To reject the signals with frequencies $f_{1}$ and $f_{2}$, an electronic analog filtering block is used. This block generates additional noise. Besides, a numerical simulation of the original method reveals that the analog filters cannot assure the necessary attenuation of the parasitic signal, when the useful signal is small.

\section{DESCRIPTION OF THE PROPOSED IMPROVED METHOD}

Fig. 1 shows a detailed block diagram of the method proposed in this paper. The first inconvenient mentioned above is overcome by using the outer and inner slots of a single chopper disk.

The light beam coming from lamp S goes through the monochromator $M$ and optical switch I1, then it is modulated in the outer section of a mechanical chopper at frequency $f_{\text {ex }}$ and shines the semi-transparent mirror $M 1(R=0,2 ; T=0,8)$.

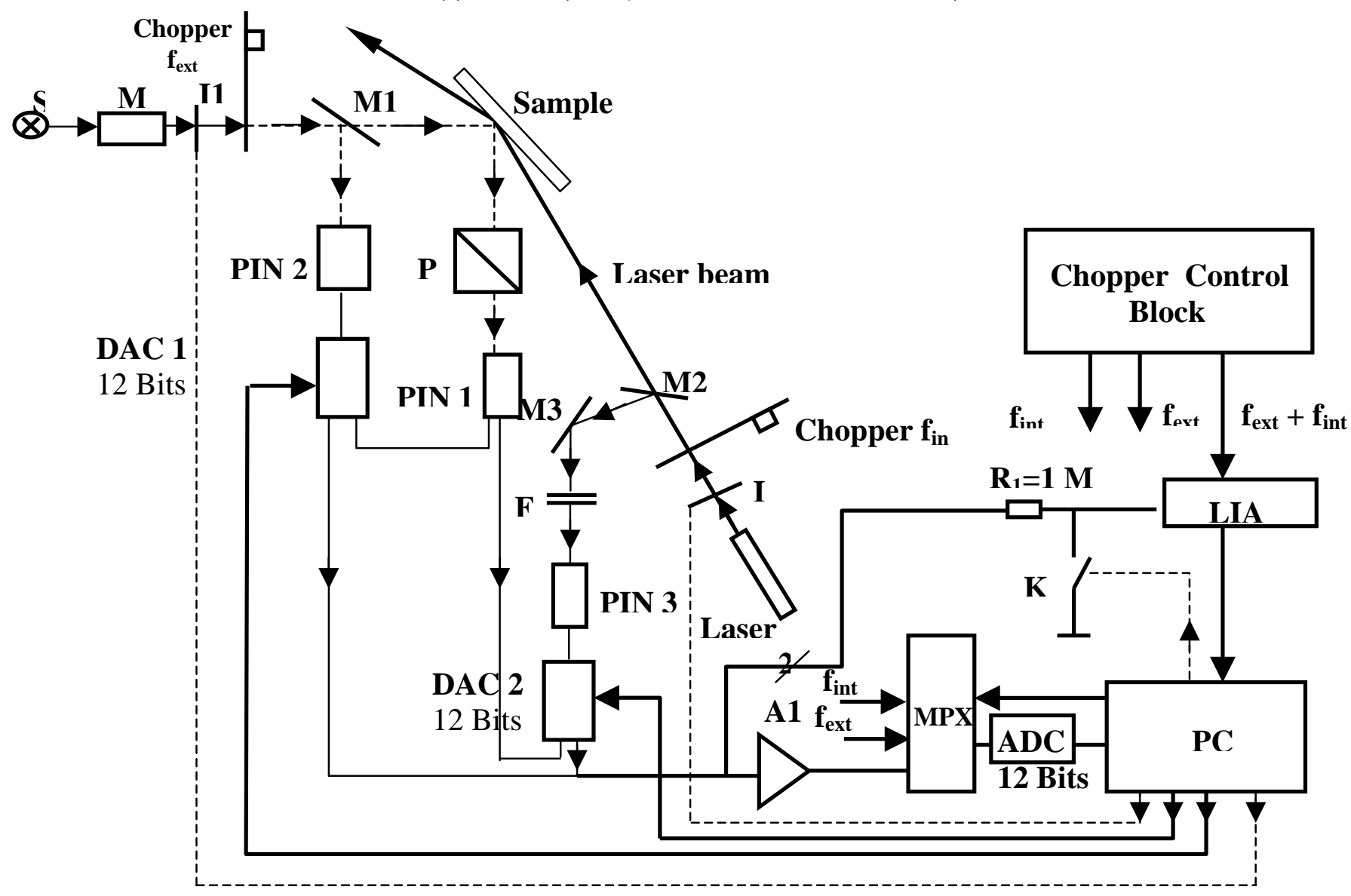

Figure 1. Block diagram of the proposed method 
The passing ray is reflected by the surface of the sample, goes trough polarizer $\mathrm{P}$ and reach the main photodiode PIN1. The reflected ray at M1, shines the compensation photodiode PIN2. The output signal of this photodiode is supplied to the reference input of DAC1, which acts as a digitally programmable attenuator.

The light beam coming from the laser goes through optical switch 12 , then it is modulated in the inner section of the same chopper at frequency $f_{\text {in }}$ and shines mirror $M 2(R=0,01 T=0,99)$. The passing ray also shines the sample. The ray, reflected by mirrors $M 2$ and $M 3$ goes through the gray filter $F(T=0,5)$ and shines the compensation photodiode PIN3. The output signal of this photodiode is attenuated in DAC2.

The three output signals of PIN1, DAC1 and DAC2 are series connected. Their polarities are such that signals from DAC1 and DAC2 are subtracted from the PIN1 signal. The resulting signal arrives through a shielded cable and the network formed by resistor $\mathrm{R} 1$ and switch $\mathrm{K}$ to the input of the lock-in amplifier. This later one is synchronized to the frequency $f_{\text {ext }}+f_{\text {in, }}$ coming from the chopper driving block. Fig. 2 shows how the connection between PIN1 and DAC1 and DAC2 can be done in practice and the polarities of the corresponding signals..

At the same time, the resulting signal is applied to the input of an amplifier with gain equal to 10 and then digitalized through channel 1 of the analog to digital converter ADC. Also the PC through channels 2 and 3, digitize the analog

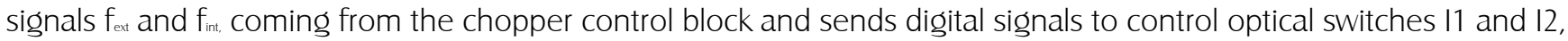
the electrical switch $\mathrm{K}$ and the attenuation value at DAC1 and DAC2.

Although in Fig. 1 with the purpose to be clearer, the ADC, the two DACS, the multiplexer and the amplifier A1 are shown as separated blocks, they can be part of a PC data acquisition board. The ADC should have a 12 bits resolution and a sampling rate equal to 500, 000 samples/sec in order to simultaneously digitize the output signal of A1 and signals $f_{\text {int }}$ and $f_{\text {ext }}$ during at least one period (5 milliseconds) and to take a sufficient number of points (100 to 500) to have an adequate representation of these signals. Equally, the DACS should have a 12-bit resolution to minimize compensation errors.

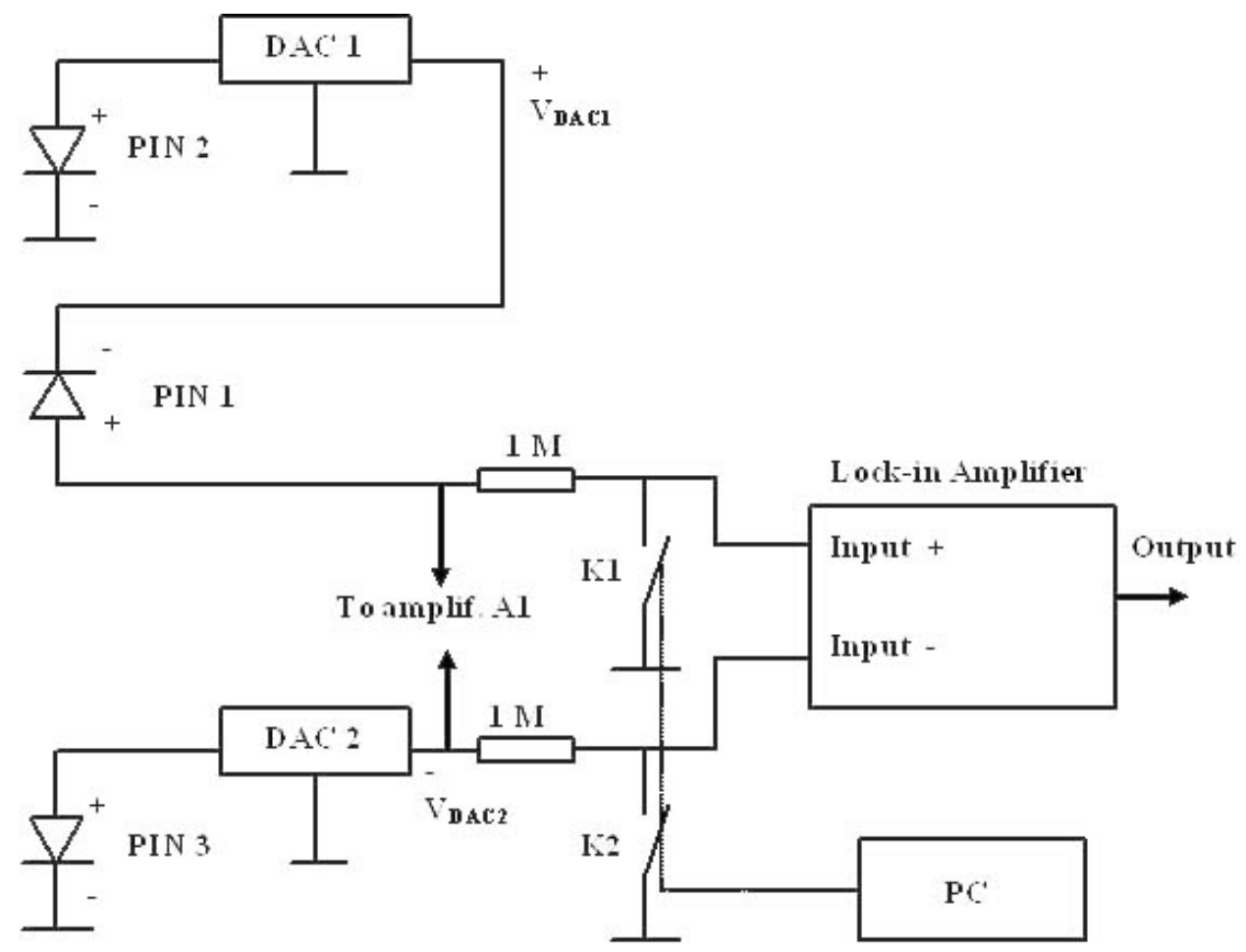

Figure 2. The connection between PIN1, DAC1 and DAC2 
A very important component of the system is the software running into the $\mathrm{PC}$, under its control the photo reflectance measurements are taken. In the following, we describe briefly the three phases of the measuring process.

\section{Phase 1: Laser scatter light compensation}

The electric switch $\mathrm{K}$ is closed to prevent the lock-in saturation due to a large signal at its input. The optical switch 11 blocks the light coming from the monochromator. The optical switch 12 is open and the laser beam goes through the chopper and mirror M2 and reaches the sample. In this way, PIN1 is solely illuminated by the laser scattered light. PIN2 is dark and therefore the output signal of DAC1 is zero. PIN3 is shinned and at the DAC2 output there is a random value. The total signal coming from the three photodiodes is not zero. This signal is supplied to amplifier A1 and digitized through channel 1 of the ADC. Simultaneously, the $\mathrm{f}_{\text {int }}$ signal is being digitized through channel 2. With both signals quantified, it is possible to implement an algorithm, which behaves like a digital lock-in. It implies filtering the $f_{\text {int }}$ signal (rectangular) to obtain a sinusoidal reference signal, multiply both signals and filtering again to obtain a useful signal free of noise. The algorithm adjusts in successive approximation steps the digital value of DAC2, in order to minimize the input signal of A1. This adjustment is done only one time for each sample and in this way the effect of the laser scattered light is compensated.

\section{Phase 2: Monochromator scattered light compensation}

The electric switch $\mathrm{K}$ is still closed. The optical switch 12 is closed and 11 opened. The beam coming from the monochromator goes through the chopper and mirror M1, reflects on the sample surface and reach PIN1. At the same time, a fraction of this beam is detoured toward PIN2. The photodiode PIN3 is dark, therefore the output signal of DAC2 is zero. The resulting signal at the A1 input is not zero. By a similar process as explained before, the A1 output and the $\mathrm{f}_{\text {ex }}$ signals are simultaneously digitized. Acting as a digital lock. in synchronized with the $\mathrm{f}_{\text {ex }}$ signal, the PC adjusts de DAC1 attenuation in order to minimize the A1 output signal. The monochromator scattered light have been compensated. This adjustment have to be done each time the wavelength is changed.

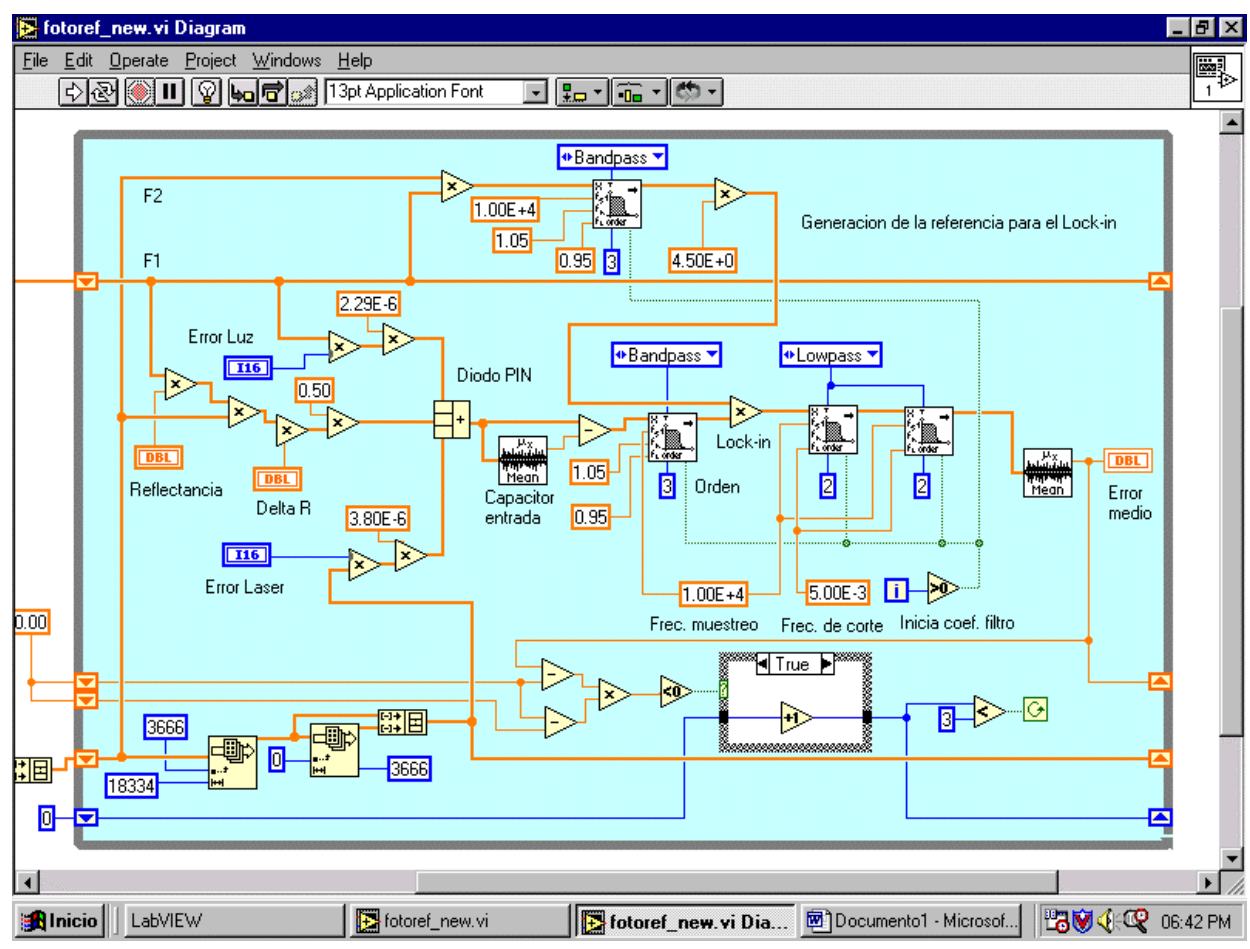

Figure 3. Lab VIEW programming window 


\section{Phase 3: Photo reflectance measurement}

The optical switches 11,12 and the electrical switch $\mathrm{K}$ are opened. The resulting signal coming from de three photodiodes PIN1, PIN2 and PIN3 reach the input of the lock-in amplifier, which is synchronized to the frequency $\mathrm{f}_{\mathrm{ext}}+$ $\mathrm{f}_{\mathrm{in}}$ coming from the chopper driving block. The digital lock-in output is sent to the PC.

As it can be seen, no further electronic block is necessary because all signals needed for synchronization are obtained from the chopper-driving block. Besides, the parasitic signals due to the laser scattered light and the monochromatormodulated light are almost compensated making unnecessary the use of any analog filtering block. The output lock-in signal corresponds to the changes in $\Delta R$, the modified reflection coefficient.

\section{NUMERICAL SIMULATION USING LABVIEW}

With the purpose to corroborate the advantages of the proposed method and its sensibility to departures from the ideal case and DACS finite resolution, a numerical simulation was carry out, using the programming language LabVIEW. Fig. 3 shows the corresponding programming screen.

In all calculations, the worst experimental case $R=0.1, \Delta R / R=10^{-5}$, laser scattered light/reflected light $=100$ was taken into account. In order to establish comparisons between different non-ideal cases and the ideal case where the laser scattered light and the monochromator-modulated light were absolutely compensated, we defined the relative error as:

$$
E_{r}=\frac{S-S_{\text {comp }}}{S_{\text {comp }}}
$$

Were $S_{\text {comp }}$ is the output signal with zero compensation error and $\mathrm{S}$ the output signal when uncompensated. During the simulation, the influence of the following three factors was investigated: 1 - The maximum compensation error of the laser scattered light and the monochromator modulated light, due to DACS finite resolution. 2- the time constants $\tau_{1}$ and $\tau_{2}$ of the lock-in low-pass filters, and 3- the dependency of the optical pulses edge duration, which can be changed by widening the laser and monochromator beams

\section{RESULTS AND DISCUSSION}

Fig. 4 shows the relative error as a function of the modulated light pulse edge duration, for several combinations of the compensation error. Axis X contains the normalized parameter edge duration/pulse duration. As it can be seen, the relative error does not depend of the pulse edge duration.

Table I shows the calculated relative errors for several combinations of compensation error, when the laser beam is modulated at frequency $\mathrm{f}_{\mathrm{nt}}$ and the monochromator at $\mathrm{f}_{\text {ext. }}$ In all cases, the relative error is smaller than $0.4 \%$.

Table II shows the results obtained when the modulation frequencies are interchanged. In this case, the relative error increase a little bit up to $0,65 \%$

In both tables, it can be seen clearly that the major contribution to the relative error comes from the monochromator beam compensation error. It is not surprising because at PIN1 active surface, the intensity of the monochromator light is too much larger than the laser light intensity. 


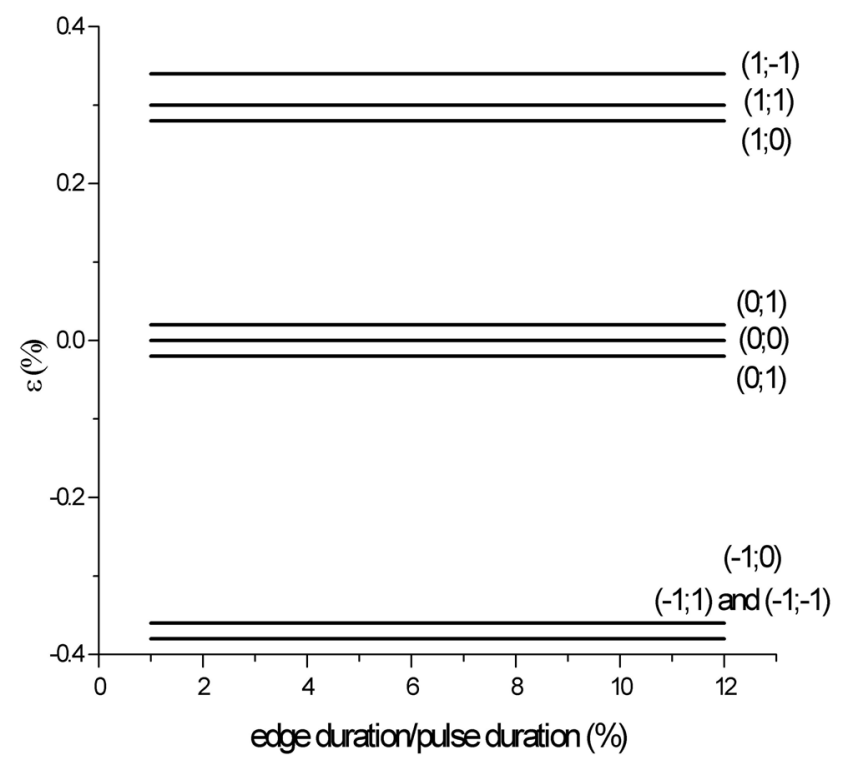

Figure 4. Relative error dependence on modulated light pulse edge duration. The numbers between the parentheses indicate monochromator scattered light compensation error:

Laser scattered light compensation error in $\angle S B$

Table I.

\begin{tabular}{|c|c|c|}
\hline \multirow{2}{*}{$\begin{array}{c}\text { Monochromator scattered light } \\
\text { compensation error }\end{array}$} & $\begin{array}{c}\text { Laser scattered light } \\
\text { compensation error }\end{array}$ & Relative error (\%) \\
\hline \multirow{3}{*}{0} & 0 & 0 \\
\cline { 2 - 3 } & 1 LSB & 0.02 \\
\cline { 2 - 3 } & -1 LSB & -0.02 \\
\hline \multirow{3}{*}{1 LSB } & 0 & 0.28 \\
\cline { 2 - 3 } & 1 LSB & 0.30 \\
\cline { 2 - 3 } & -1 LSB & 0.34 \\
\hline \multirow{3}{*}{-1 LSB } & 0 & -0.36 \\
\cline { 2 - 3 } & 1 LSB & -0.38 \\
\cline { 2 - 3 } & -1 LSB & -0.38 \\
\hline
\end{tabular}

Settings: $\tau 1=\tau 2=0.1 \mathrm{sec}$, Monochromator light modulated at fext $=240 \mathrm{~Hz}$. Laser light modulated at fint $=200 \mathrm{~Hz}$

Fig. 5 shows the relative error dependency on the time constant $\tau_{1}$ of the lock-in first low-pass filter, for extreme values of the compensation errors of both beams.

The light coming from the monochromator is modulated in the outer section of the chopper disk at a frequency $\mathrm{f}_{\text {ext }}=$ 240 Hertz, while the laser light is modulated in the inner section of the disk at a frequency fint $=200$ Hertz. The time constant $\tau_{2}$ of the lock-in second low-pas filter was kept constant and equal to $0.1 \mathrm{sec}$ for simplicity at simulation time (the other option, $\tau_{2}=1 \mathrm{sec}$ isn't practical, because it increase to much the measuring time) 
Table II.

\begin{tabular}{|c|c|c|}
\hline $\begin{array}{c}\text { Laser scattered light } \\
\text { compensation error }\end{array}$ & $\begin{array}{c}\text { Monocromator scattered } \\
\text { light compensation error }\end{array}$ & Relative error (\%) \\
\hline \multirow{3}{*}{0} & 0 & 0 \\
\cline { 2 - 3 } & 1 LSB & 0.02 \\
\cline { 2 - 3 } & -1 LSB & -0.02 \\
\hline \multirow{3}{*}{1 LSB } & 0 & 0.60 \\
\cline { 2 - 3 } & 1 LSB & 0.60 \\
\cline { 2 - 3 } & -1 LSB & 0.60 \\
\hline \multirow{3}{*}{-1 LSB } & 0 & -0.60 \\
\cline { 2 - 3 } & 1 LSB & -0.65 \\
\cline { 2 - 3 } & -1 LSB & -0.60 \\
\hline
\end{tabular}

Settings: $\tau 1=\tau 2=0.1 \mathrm{sec}$. Laser light modulated at fext $=240 \mathrm{~Hz}$. Monochromator light modulated at fint $=200 \mathrm{~Hz}$

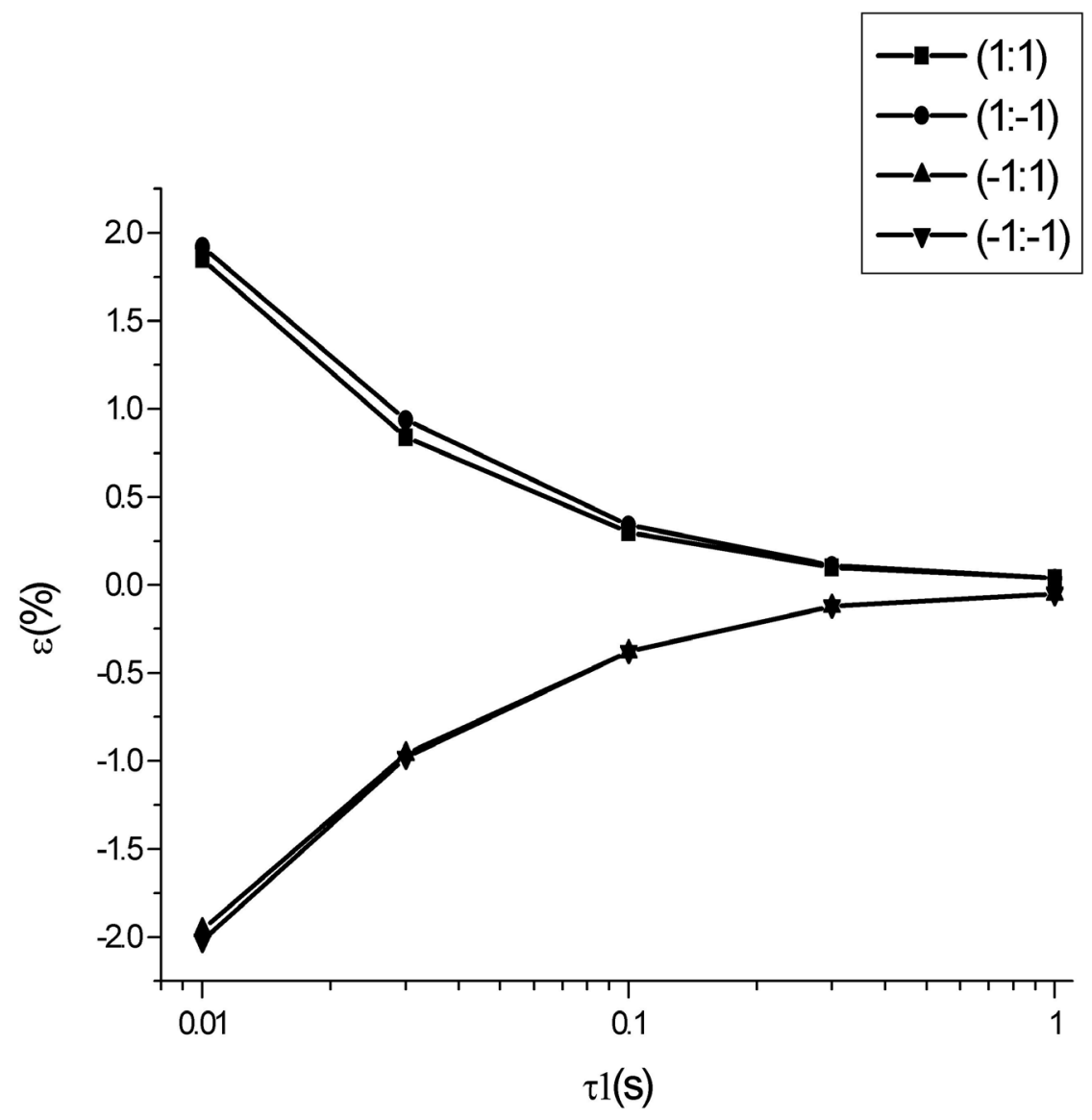

Figure 5. Relative error dependence on the time constant $\tau 1$ of the lock-in first low-pass filter.

The numbers Between the parentheses indicate (monochromator scattered light compensation error; Laser scattered Light compensation error) in LSB 


\section{CONCLUSIONS AND RECOMMENDATIONS}

From the above mentioned results, we can derive the following conclusions:

1. It seems that using the proposed method, a relative error smaller than $1 \%$ is attainable, even in the experimental worst case $\left(\mathrm{R}=0.1 ; \quad \Delta \mathrm{R} / \mathrm{R}=10^{5}\right)$.

2. The relative error doesn't depend on the light pulses edge duration. This is a good new, because it eliminates the need of precise adjustment of these parameters.

3. The relative error almost doesn't depend of time constants $\tau_{1}$ and $\tau_{2}$ of the lock-in low-pass filters, if they are grater than $0,1 \mathrm{~s}$ and when the modulation frequency is greater than 240 Hertz.

For this reasons, it is advisable to use a regime where the monochromator beam was modulated in the outer chopper section at $f_{\text {ext }}=240$ Hertz and the laser beam in the inner section at $f_{\text {int }}=200$ Hertz. The lock-in low-pass filters time constants should be adjusted to $\tau_{1}=\tau_{2}=0.1 \mathrm{sec}$

\section{REFERENCES}

[1] Shen H., Dutta M. "Sweeping photoreflectance spectroscopy of semiconductors", Appl. Phys. Lett., 57, (6), 587, (1990)

[2] Sousa D.F., Bell M.J.V., Nunes L.A.O., "Photoreflectance and time-sesolved photoreflectance in delta-doped superlattices", J. Appl. Phys., 83, (5), 2806, (1998)

[3] Sydor M., Badakshan A., "Different photoreflectance from a high-mobility and highly luminescent twodimensional electron gas", J. Appl. Phys., 70, (4), 2322, (1991)

[4] Klem J.F., Breiland W.G., Fritz I.J., Drummond T.J., Lee S.R., " Application of in situ reflectance monitoring to molecular beam epitaxy of vertical-cavity structures", J. Vac. Sci. Technol., B16 (3) 1498, (1998)

[5] Ghosh S., Arora B.M., "Photo reflectance spectroscopy with white light pump beam", Rev. Sci. Instrum., 69, (3), 1261, (1998)

[6] Catalog "Scientific and Engineering Instruments", Ed.: "Stanford Research Systems", 84, (2000)

Authors Biography

\section{Manuel Hernández Calviño}

Physics Major at Havana University (1970), Master in Science in Microelectronics (1975), Doctor on Physics (1980). Full professor of Electronics at Faculty of Physics, Havana University, since 1970. He has 51 papers presented in international congresses in Cuba, Spain and México, 32 papers published in specialized journals in Cuba, Spain and México, and two books and several laboratory manuals published and devoted to teaching Digital and Analog Electronics. He has taught 28 postgrade courses at Havana University, Universidad Iberoamericana de la Rábida and Universidad de Córdoba Spain and UAZ México. Holds two patent certificates. Founder member of the Cuban Society of Physics, Member of the IEEE Journal of Electron Devices. IEEE Senior Member.

\section{Rumen Ivanov Tsonchev}

Was born in Sofia, Bulgaria, in 1952. He received the M. Sc. Degree in Electronics and Radiophysics from the University of Sofia, Bulgaria, in 1977, and the Ph D. degree in Biomecanics from the Bulgarian Academy of Sciences in 1989. From 1977 to 1998, he was a Research Assistant at Medical University in Varna, Bulgaria. Since 1998, he has been Research Profesor in the Faculty of Physics of the Independent University of Zacatecas, México. His primary interests are measuring techniques in Physics and Medicine. He is the author co-author of about scientific papers. 